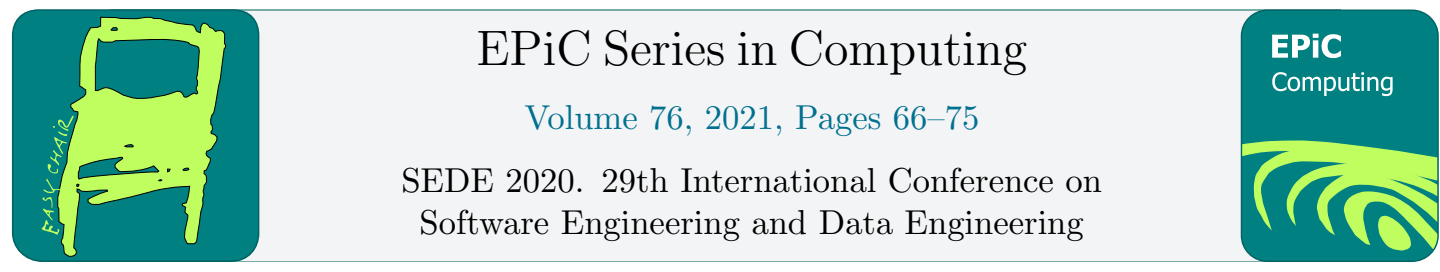

\title{
Techniques for Using Virtual Reality Simulations for Self-Defense Skill Development
}

\author{
John Apo and Alexander Redei \\ Department of Computer Science, Central Michigan University \\ Mount Pleasant, Michigan \\ (apo1j, redei1a)@cmich.edu
}

\begin{abstract}
KickVR is a training simulation designed to teach self-defense. With realistic training and a user-friendly guide, its aim is to help users learn how to better defend themselves in the real-world. Although the chances of being in a scenario where one may need selfdefense are relatively low, self-defense is an important skill to have. The goal is to enable users to learn self-defense even if they do not have the time to attend in-person classes or do not have self-defense classes in their region. Virtual reality provides convenient access to self-defense training for users in their own homes. It also provides the feeling of realism by implementing the use of hand-tracking. The user's real-world hands are rendered for the self-defense simulation rather than using Oculus hand controllers. This document will cover the current implemented features of KickVR and offer suggestions on areas of improvement based on an internal and external evaluation of software limitations.
\end{abstract}

\section{Introduction}

Central Michigan University made national headlines in February 2018 when a 19-year-old student shot and killed his parents in his dorm room during spring break move out causing the campus to go on lock-down [1]. More recently, in February 2020, a 19-year-old freshman student at Central Michigan University went to Wayside Central, the local night club, on a Saturday night and stabbed three attendees during an altercation [2]. These events seemed significant at the time, but crime has become increasingly more common and more likely to occur in this area. NeighborhoodScout ranks Mount Pleasant, Michigan as a 29 out 100 on safety, meaning Mount Pleasant is safer than only 29 percent of cities in the United States with roughly 20 crimes happening per 1,000 people per year. With nearly 15,000 students taking on-campus classes in Fall 2019, that equates to nearly 300 students becoming victims to some sort of crime during the academic year [3]. Although property crimes are lower than the national average, a Mount Pleasant resident is twice as likely to be murdered, four times as likely to be raped, and seven times as likely to be robbed than the average United States citizen [4].

While crime rates have substantially decreased in over the past two decades a study found many victims are not reporting crimes, according to the study, as many as two-thirds of crimes may be going unreported [5]. To continue to lower crime rates and empower victims of crime 
who do not feel confident reporting crimes to police, a self-defense class in virtual reality, dubbed KickVR, was created to simulate scenarios where self-defense might be necessary. When conflict resolution skills are not sufficient in deescalating a conflict, successfully employing self-defense techniques is necessary to protect vulnerable populations like young women. One study at the University of Oregon found positive benefits for female college students who completed a self-defense class [6]. In fact, a national self-defense program which has had over 60,000 people complete their class reported that 97 percent of their graduates were able to fight off their attacker [7].

Using these programs as a model for the ideal self-defense class, we partnered with the Mount Pleasant Police Department to identify aspects of self-defense training that would contribute to successful skill development. Police Officer Justin Nau recommended teaching Krav Maga fighting techniques as they are the techniques that are using in many police and military organizations and are currently taught to sororities that opt-in to training offered by the Mount Pleasant Police Department. He also identified high-risk areas for crime in Mount Pleasant that we could virtually recreate including the local bar called O'Kelly's Sports Bar \& Grill, Main Street which hosts a large majority of Central Michigan University's fraternities and sororities, and walking back to the car at night from the library or grocery store. These scenarios were generalized for a wider audience to use for skill development nationwide in hopes that anybody could download KickVR and learn skill development to defend themselves or assist others from becoming victims of violence.

\section{Background}

While there are many self-defense resources available over the Internet, none combine the educational, realism, and entertainment components of KickVR. There are plenty of services that offer self-defense training such as free videos on social media platforms like YouTube while other mediums come at a price such as physical classes. KickVR combines the benefits of both types of services. With a virtual reality self-defense class, consumers can train through self-paced realistic simulations in the comfort of their own home. Training through the simulation is available 24/7 and users get better, more interactive practice than what passive videos provides. KickVR brings the defense class into living rooms nationwide and help train users to better protect themselves while keeping them in their comfort zone. A survey of our peers found that interest is high in a product like KickVR since self-defense videos garner millions of views and there are many self-defense facilities located around the world. Classes can also be very expensive whereas this product might have a one-time smaller fee similar to a video game or could be a free product.

Video game marketplaces like Steam feature many self-defense games, but the only similar self defense class that combines gaming and education would be Self-Defense Training Camp, a Microsoft Kinect title that puts players in specific situations and has them mimic the actions on screen to progress through the game. Other video games like Creed: Rise to Glory and Drunkn Bar Fight seen in Figure 1 bridge the education and video gaming gap, but miss key elements. Creed features realistic boxing combat and boxing technique training, but the goal of the game is to fight for sport and competition. In a real scenario, there are no regulations and someone who is vulnerable needs to be able to protect themselves and counter attacks with every resource available when deescalation is not possible. Drunkn Bar Fight features realistic combat through ragdoll physics and grabbing items in the environment to fight with, but does not teach the user how to properly employ self-defense techniques, solely focusing on video game entertainment. The primary competition are the real physical classes such as Spartan 
Krav Maga [8] that teach self-defense, and videos that teach self-defense techniques over the Internet. Virtual reality games could also act as secondary competition since this product doubles as a game that is enjoyable to play while informative about self-defense.
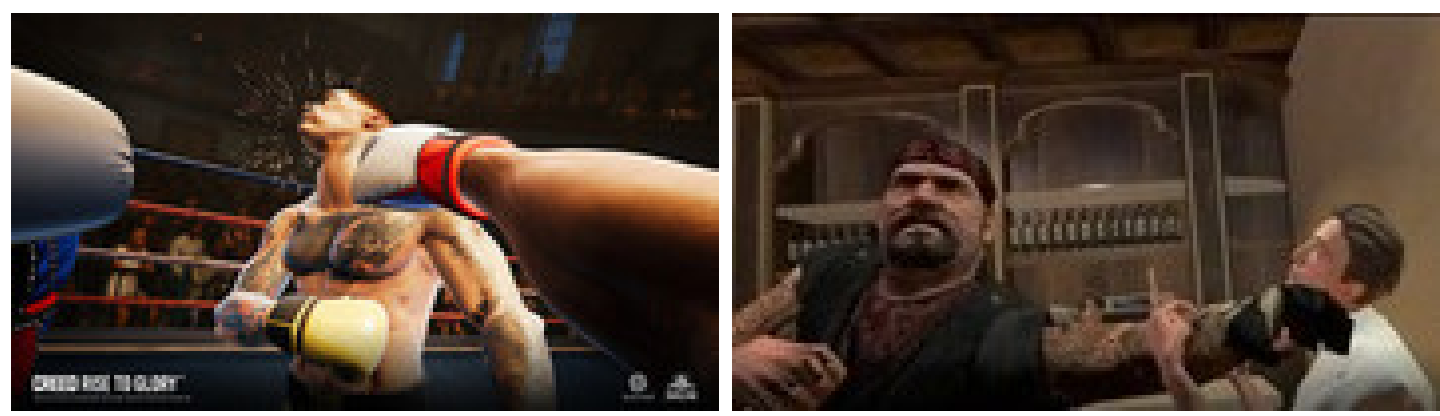

Figure 1: Screenshots of Creed: Rise to Glory [9] (left) and Drunkn Bar Fight [10] (right)

On the other hand, self-defense videos are easy to access and can be found online for free. However, they lack real practice or simulation. Viewers might practice the moves shown on screen, but without a scrimmage partner or accountability from an instructor simulating the technique in a realistic scenario, the technique is not developed properly. Physical classes are great for this reason as you can practice the moves taught to participants on other students or the instructor. It can show the user how these self-defense techniques actually work in the real world and let the participant truly practice them. However, classes can be expensive, require participants to form a schedule around them, show up in person which is difficult in the wake of COVID-19, and can be intimidating for some people, especially those who rarely step foot in a gym or dojo. Our virtual reality self-defense class will let the user choose when to train, and from the comfort of your own home, just like a video. Then, similar to a traditional class, users can practice real-world scenarios through the immersive virtual world. In the end, the virtual reality self-defense simulation doubles as a fun video game and a potentially life-saving course.

Unity and Epic Games' Unreal Engine make virtual reality simulation development possible for most developers. Since Central Michigan University teaches Unity development and has resources and faculty members trained for Unity, KickVR was created in Unity. Unity offers many advantages for VR development including a high definition render pipeline for realistic graphics, spatial audio for positional sounds in the virtual space, and most importantly the ability to build the application for multiple platforms including Android, iOS, Windows, HTC Vive and Oculus Rift [11].

\section{System Design}

KickVR is a virtual reality simulation, which is "a simulated experience that can be similar to or completely different from the real world" [12]. In this situation, the virtual reality simulation is meant to be as real as possible to master a difficult, potentially life-threatening task in a safe environment. During the creation of KickVR, it was easy to access resources necessary to develop the virtual reality simulation, but it was difficult to access the technology required to run and test the simulation. The Oculus Rift requires the user to have the following minimum system requirements: Intel i3-6100 / AMD Ryzen 3 1200, FX4350 or greater processor, NVIDIA GTX $1050 \mathrm{Ti}$ / AMD Radeon RX 470 or greater graphic card, and 8GB+ RAM. However, 
to maximize the graphics and processing speed of the Oculus Rift, the user must have the recommended system requirements: Intel i5-4590 / AMD Ryzen 5 1500X or greater processor, NVIDIA GTX 1060 / AMD Radeon RX 480 or greater graphics card, and 8GB+ RAM [13].

By default, the first generation of Oculus Rift headsets comes with two hand controllers. While they are easily tracked within the play-space the user sets up through the Oculus' Guardian System, it reduces the immersion and realism of the simulation. In a real-world situation where self-defense may be necessary, the user will not have hand controllers. The absence of hand controllers in combination with quality, supported hardware systems will allow for deeper immersion and a more quality experience. Instead, KickVR utilizes a Leap Motion Controller mounted on the Oculus headset which tracks the movement of the user's hands in real life to project in the simulation. One limitation of the Leap Motion Controller is that tracking is very limited to only in front of the user's headset. This was an acceptable limitation for this simulation since most self-defense moves are meant to be performed head-on and only used enough to incapacitate or escape the conflict.

KickVR also uses applicable, real-life scenarios with instructions from certified Krav Maga experts to guide the users to successfully learn self-defense techniques. The user interface is simple but informative as to not interfere with immersion. Additionally, there are several modules of varying difficulty levels and skill development objectives. The easier modules were developed first with more difficult modules being added incrementally. The module will be comprised of a training portion, an application scenario, and an evaluation of skill development upon completion of the training module with short statistics on that scenario in American society to further enrich and motivate skill development as seen in Figure 2.

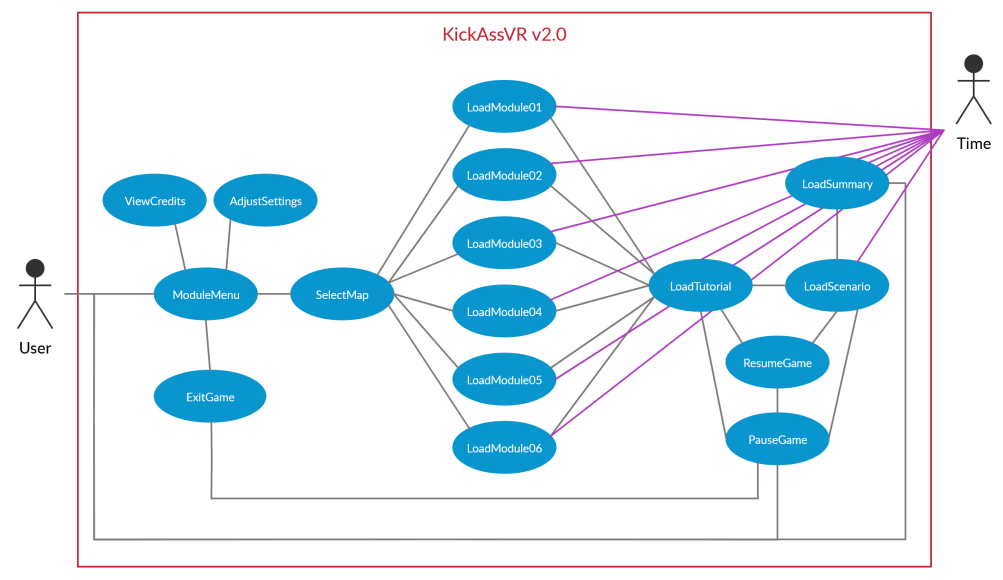

Figure 2: Use Case Diagram for KickVR

The following technical requirements added or being added to KickVR is shown in Table 2 $\&$ Table 3. The priority of these requirements is described in Table 1. 
Table 1: Requirement Priority

\begin{tabular}{|c|l|}
\hline Priority & Description \\
\hline 1 & Denotes requirements we must implement by May 2020. \\
2 & Denotes requirements we should implement by December 2020. \\
3 & Denotes requirements we may implement if time allows. \\
\hline
\end{tabular}

Table 2: Functional Requirements

\begin{tabular}{|c|c|c|}
\hline Requirement & Priority & Description \\
\hline FR01 & 1 & The simulation must initially load up to a start screen or main menu. \\
\hline FR02 & 1 & The user must be able to exit the simulation. \\
\hline FR03 & 1 & $\begin{array}{l}\text { The simulation must have a menu from which the user can select train- } \\
\text { ing modules. }\end{array}$ \\
\hline FR04 & 1 & The user must be able to control a character in each training module. \\
\hline FR05 & 1 & $\begin{array}{l}\text { The user must be able to receive directions in each training module via } \\
\text { signposting and colored visual cues. }\end{array}$ \\
\hline FR06 & 1 & $\begin{array}{l}\text { The simulation must provide a tutorial and an application in each train- } \\
\text { ing module. }\end{array}$ \\
\hline FR07 & 1 & $\begin{array}{l}\text { The user must receive additional information on that module's self- } \\
\text { defense technique or a statistic related to self-defense. }\end{array}$ \\
\hline FR08 & 1 & $\begin{array}{l}\text { The user must be able to see their physical hands projected virtually } \\
\text { in the simulation. }\end{array}$ \\
\hline FR09 & 1 & The simulation must have a tutorial training module. \\
\hline FR10 & 1 & $\begin{array}{l}\text { The simulation must have an open-hand strike or punch training mod- } \\
\text { ule. }\end{array}$ \\
\hline FR11 & 1 & $\begin{array}{l}\text { The simulation must have an acknowledgment list to give credit to its } \\
\text { authors, advisors, and resources. }\end{array}$ \\
\hline FR12 & 2 & $\begin{array}{l}\text { The simulation should have sound effects and haptic feedback in pe- } \\
\text { ripherals to enhance training. }\end{array}$ \\
\hline FR13 & 2 & The user should be able to pause and resume their training modules. \\
\hline FR14 & 2 & The simulation should have a closed-hand strike training module. \\
\hline FR15 & 2 & The simulation should have an elbow strike training module. \\
\hline FR16 & 2 & $\begin{array}{l}\text { The simulation should grade the user on the effectiveness of their self- } \\
\text { defense techniques at the end of each training module. }\end{array}$ \\
\hline FR17 & 3 & $\begin{array}{l}\text { The user may be able to select the environment they would like to test } \\
\text { their skills in during the application portion of their training module. }\end{array}$ \\
\hline FR18 & 3 & The simulation may have a grappling training module. \\
\hline FR19 & 3 & The simulation may have a knee strike training module. \\
\hline FR20 & 3 & The simulation may have background music samples. \\
\hline FR21 & 3 & The simulation may have accessibility options to adjust the volume. \\
\hline FR22 & 3 & The simulation may feature an endless survival mode. \\
\hline FR23 & 3 & The simulation may feature interactive, physics-driven objects. \\
\hline
\end{tabular}


Table 3: Non-Functional Requirements

\begin{tabular}{|c|c|c|}
\hline Requirement & Priority & Description \\
\hline NFR01 & 1 & The simulation must be playable on Windows platforms. \\
\hline NFR02 & 1 & The simulation must be developed in Unity. \\
\hline NFR03 & 1 & $\begin{array}{l}\text { The simulation must be compatible with the Oculus Rift/Quest virtual } \\
\text { reality system and peripherals. }\end{array}$ \\
\hline NFR04 & 1 & The simulation must be compatible with the Leap Motion Controller. \\
\hline NFR05 & 1 & The simulation must have a functional, aesthetic user interface. \\
\hline NFR06 & 2 & The latency for loading scenes should not exceed 30 seconds. \\
\hline NFR07 & 2 & $\begin{array}{l}\text { The simulation should have a frame rate of at least } 30 \text { frames per second } \\
\text { to prevent virtual reality sickness. }\end{array}$ \\
\hline NFR08 & 3 & The simulation may be used and enhanced with a haptic vest. \\
\hline NFR09 & 3 & The simulation may be playable on MacOS platforms. \\
\hline
\end{tabular}

\section{User Experience}

Hand tracking and hand gestures triggering logic in the simulation are one of the most impressive features of KickVR. Using a Leap Motion Controller mounted to the front of an Oculus Rift headset, key points like joints and the palm of the hand are found in real life and those positions are replicated in the virtual world. A hand mesh is then projected on those points to make the hand appear in the simulation as seen in Figure 3. Latency between hand movements in real life versus the virtual world were a concern while developing KickVR because small delays visualization of movements can cause disorientation and motion sickness. Many factors play into response time of hand tracking including hardware, software, and graphics constraints [14]. The Leap Motion Controller is configured to track a user's hands using three different settings: High-Speed Mode, Balanced Mode, and Precision Mode. High-speed mode focuses on maximizing frames per second while minimizing resolution which is good for games like Drunkn Bar Fight where graphics are not necessary for quality game-play. On the other hand, precision mode focuses on precise hand movements at the expense of frame rate which would be important for simulations that may require the user to interact with objects in the environment. Balanced mode is the middle ground between the two modes and is the default scanning mode.

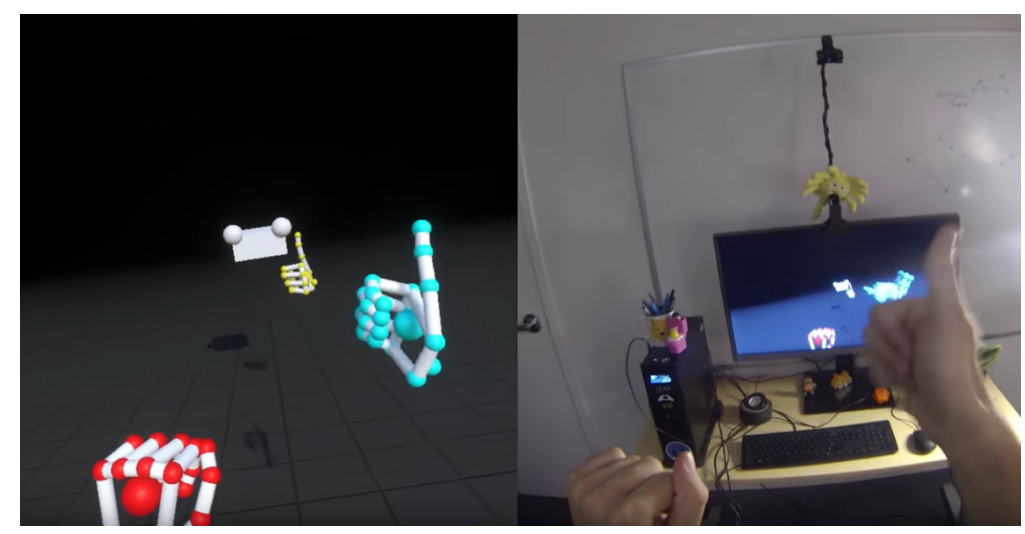

Figure 3: A screenshot of Leap Motion developers using hand tracking from Leap Motion's Unity Core Assets[15] 
At the hardware level, there could be as much as a 15 millisecond time difference between using a USB 2.0 versus USB 3.0 port for data transferring [14]. At the software level, the Leap Motion client on the user's PC handles all processing of data transferred from the USB port and cannot be sped up from the user's perspective. For visualization, latency can be affected by hardware components such as operating system, graphics card, monitor resolution, and physical circuitry of the monitor [14]. The average total processing time is around 60-70 milliseconds since the Leap Motion Controller only uses USB 2.0 speeds [16] which is satisfactory to prevent motion sickness. More settings can be changed to optimize latency including disabling vertical synchronization in graphics card settings and using a higher refresh rate monitor [16].

When the user configures the Leap Motion Controller, configures the Oculus Rift, and executes the simulation, the user loads into the virtual hub which acts as a three-dimensional main menu. In this scene, the user can choose which training module and thus which self-defense technique they would be interested in learning and can select an environment they would like to apply their skills in as seen in Figure 4.
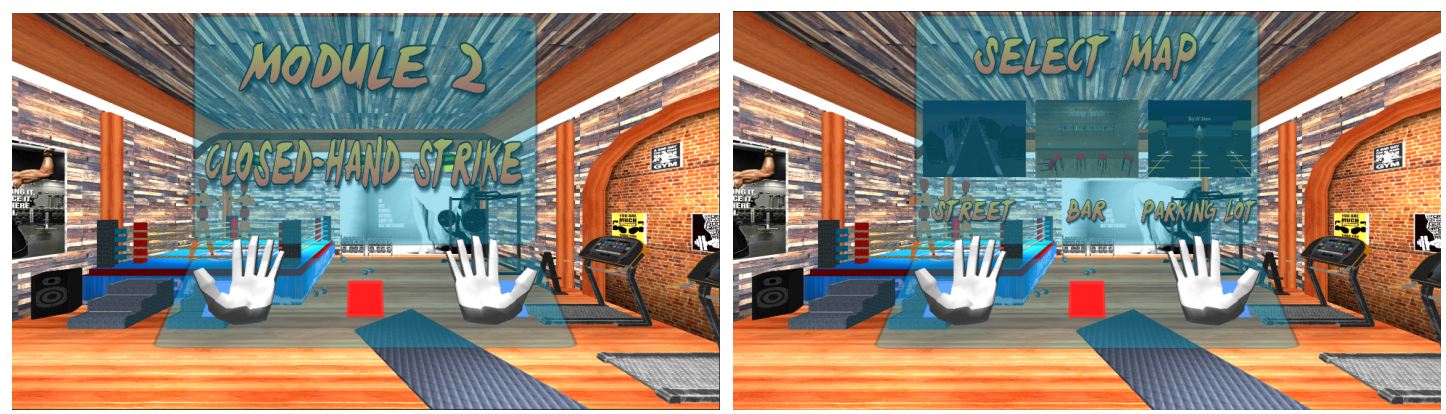

Figure 4: Module menu (left) and map selection (right) in the virtual hub in KickVR

In each training module, the user completes two segments: a skill development and a skill application segment. The skill development portion is completed first which can be seen in Figure 5. The user will learn that module's skill from a certified Krav Maga expert via video and be able to endlessly practice those skills in a safe gym environment. When they are ready to move on, they use the Leap Motion's hand gestures which take them to the skill application environment they chose in the virtual hub.
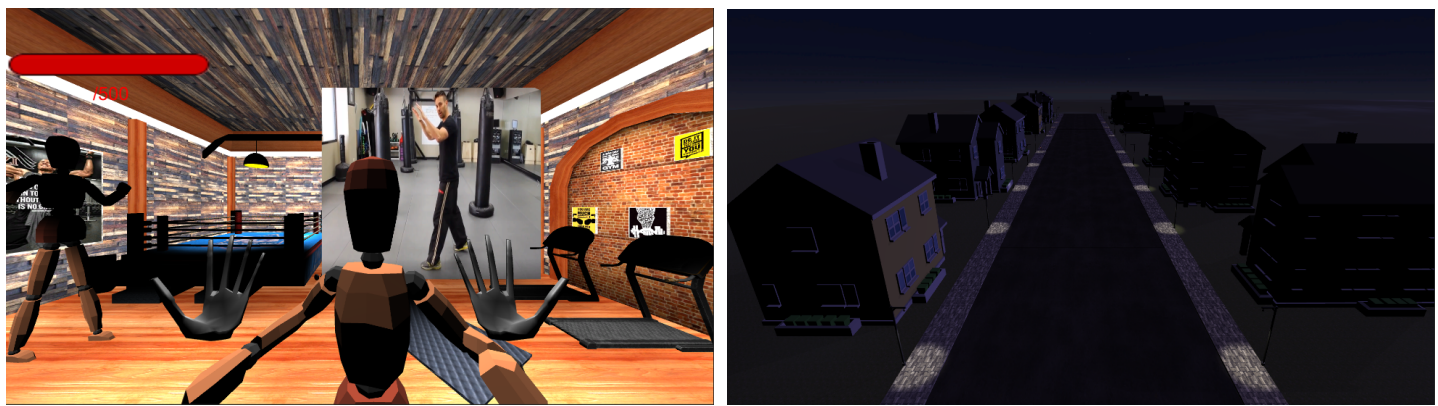

Figure 5: Training environment in the tutorial module (left) and street scenario (right) in KickVR 
There are three environments as seen in Figures 5 and 6: a street scenario at night, a store parking lot scenario at night, and an indoor bar scenario. Upon loading into the scenario, the NPC loops through fighting animations such as punching, guarding, and elbowing. The user applies their fighting skills from that training module and previously completed scenarios. The user is meant to guard themselves from incoming attacks and also attack the NPC when they are vulnerable to a counter attack. The skill application segment ends when the user or NPC loses all of their health. From there, the user is taken to a summary scene which is seen in Figure 7. In this scene, the user can understand all hits they dealt and received via a scoreboard. The point tally is meant to show efficiency of skill application and skill mastery. Higher scores indicate that the user more efficiently utilized their self-defense skills.
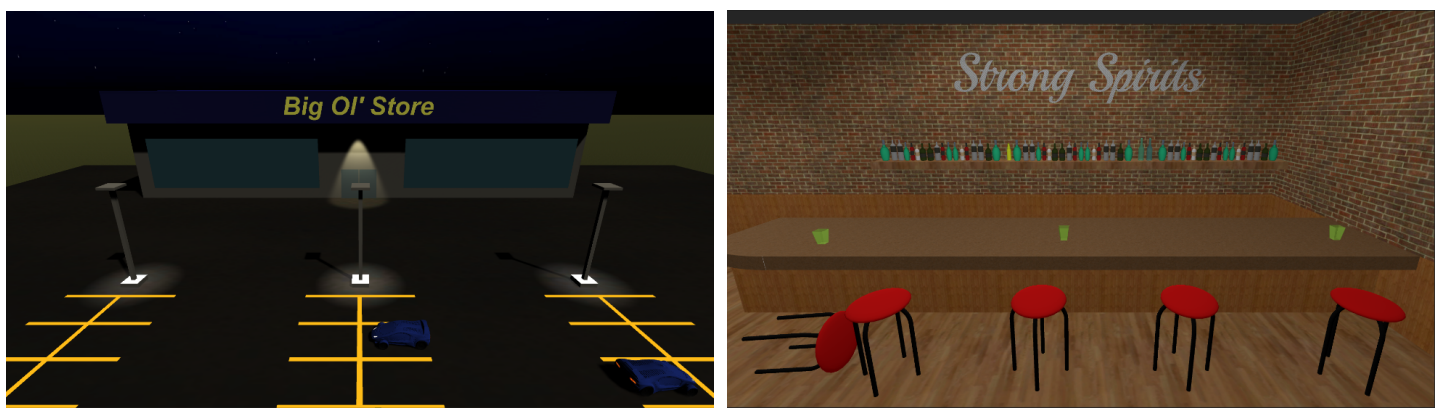

Figure 6: Store parking lot scenario (left) and bar scenario (right) in KickVR

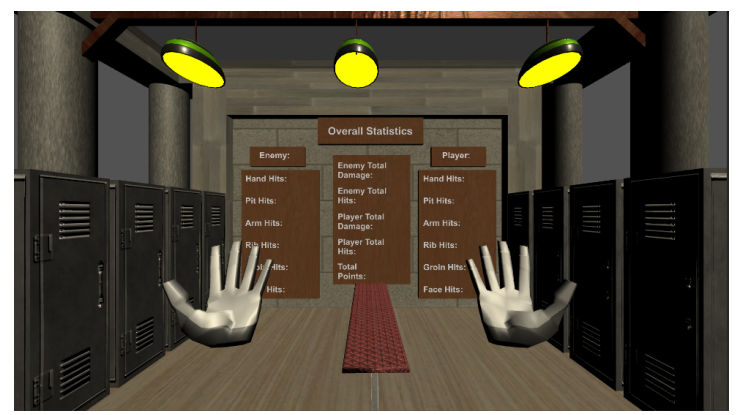

Figure 7: Skill development summary scene in KickVR

The efficiency score is computed via the trigger areas and colliders on the NPC and user models as seen in Figure 8. Trigger areas call scripts when they are interacted with. Colliders, which are slightly smaller than trigger areas, are used as a barrier so models, like the user's hands, do not visually clip into other models, like the NPC's body. Each collider has one trigger. The user has one body and two hand colliders. The NPC model has several colliders including two hand, two arm, one abdomen, one chest, one groin, and one head collider with respective trigger areas. Unless blocked by the user's hand colliders, the NPC deals 50 damage per hit to the user. When a collider on the NPC is struck by the user's hand, a collider script determines which on the NPC was struck and increments the respective counter to deduct NPC health. For example, if the user strikes the NPC in the head, it will result in an 80-point decrement of NPC health. Since the head is the most vulnerable part of the human body, which the user 


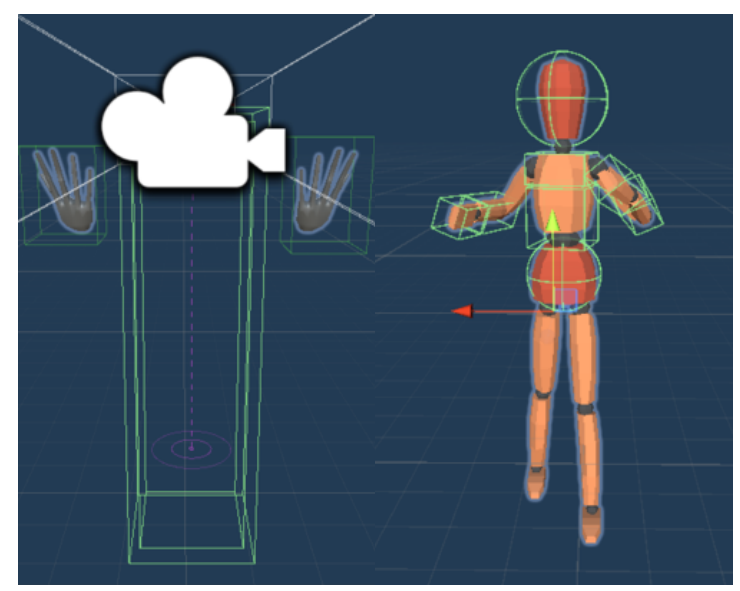

Figure 8: User colliders (left) and NPC colliders (right)

learns in the tutorial training module, 80 damage is the most damage the user can deal with damage scaling down for each body part with the minimum damage dealt being 10 if the user hits the NPC's hands.

\section{Future Work and Conclusion}

In this paper, we discussed the motivation for creating KickVR, the development process, and its strengths and limitations. In addition, we reviewed the hand-tracking capabilities of the software and offered some insight into how virtual reality simulations can be used for training. We strongly feel that self-defense skill development should be more prioritized in society. In addition to the physical benefits of self-defense training, it also provides many psychological benefits. One preliminary study found that self-defense training produced increases in self-esteem, perceived control, and confidence while decreasing anxiety, fear, and avoidance behaviors in women who took a self-defense class [17]. KickVR can satisfy both self-defense competency needs and psychological safety needs.

For future work, we are interested in adding features such as new training modules and new training application environments like the inside of a house. In addition, building an Oculus Quest-compatible simulation is a major priority for better hand-tracking. Hand-tracking was the most difficult part of developing KickVR because of the need to integrate communications between two hardware devices. The Leap Motion Controller was also prone to visual glitches or lack of accuracy due to smudging on the sensor or competitive calls to the CPU. In December 2019, Oculus announced that they have released in-house hand-tracking capabilities for the Oculus Quest through a software update [18]. This reduces overhead price of running KickVR for consumers since there would be no need to purchase a Leap Motion Controller and it would reduce overhead processing power necessary to run both the Oculus Rift and Leap Motion accessories simultaneously. This could be implemented by updating the Oculus Integration asset package in Unity and replacing the Leap Motion hand-tracking GameObjects and script logic with the respective Oculus Integration hand-tracking GameObjects.

To increase realism, we are also interested in implementing inverse kinematics (IK) through Unity's physics engine. IK enables the NPC to be effected by a force applied by the user's hand 
via a punch. For example, a punch to the left side of the NPC's head would result in the NPC's head moving right while not effecting any script logic. We hope to participate in and demo these features at the Great Lakes Bay Research Consortium in August and eventually publish KickVR on Steam for widespread public use.

\section{References}

[1] L. Wamsley, V. Romo, and P. Shah, "Suspect in custody after deadly shooting at central michigan university," NPR, Mar 2018. [Online]. Available: https://www.npr.org/sections/thetwo-way/ 2018/03/02/590239044/central-michigan-university-on-lockdown-after-shooting-at-dorm-kills-2

[2] B. Parvez, "19-year-old freshman charged after 3 stabbed at bar near central michigan university," Detroit Free Press, Feb 2020. [Online]. Available: https://www.freep.com/story/news/local/michigan/2020/02/25/ central-michigan- university-freshman-arraigned-stabbing-wayside-bar/4865968002/

[3] C. M. University, "On-campus student profile fall 2019," 2019 Student Enrollment Statistics, Aug 2019. [Online]. Available: $\quad$ https://www.cmich.edu/office_provost/academic_administration/APA/Reports/ Documents/FallStudentEnrollmentProfiles/On_campus/on_campus_student_fall_profile_2019.pdf

[4] "Mount pleasant, mi crime rates," n.d. [Online]. Available: https://www.neighborhoodscout. $\mathrm{com} / \mathrm{mi} / \mathrm{mount}$-pleasant/crime

[5] J. Gramlich, "5 facts about crime in the u.s." Pew Research Center, Oct 2019. [Online]. Available: https://www.pewresearch.org/fact-tank/2019/10/17/facts-about-crime-in-the-u-s/

[6] L. Raleigh, "Are women safer when they learn self-defense?" Cascade Magazine, 2013. [Online]. Available: https://cascade.uoregon.edu/spring2013/social-sciences/ are-women-safer-when-they-learn-self-defense/

[7] "Success rate of graduates fighting back," n.d. [Online]. Available: http://modelmugging.org/ success-rate-of-graduates-fighting-back/

[8] "Spartan krav maga store," Oct 2016. [Online]. Available: https://www.spartankravmaga.com/ store/

[9] Survios, "Creed: Rise to glory ${ }^{\top M}$ on steam," Steam, Sep 2018. [Online]. Available: https://store.steampowered.com/app/804490/Creed_Rise_to_Glory/

[10] T. Munky, "Drunkn bar fight on steam," Nov 2016. [Online]. Available: https: //store.steampowered.com/app/528550/Drunkn_Bar_Fight/

[11] "Virtual reality," n.d. [Online]. Available: https://unity.com/unity/features/vr

[12] B. Pursel, "Chapter 10: Design methodologies," in Information, People, and Technology. Pennsylvania State University, 2005. [Online]. Available: https://psu.pb.unizin.org/ist110/ chapter/6-3-virtual-reality/

[13] "Oculus rift and rift s minimum requirements and system specifications," n.d. [Online]. Available: https://support.oculus.com/248749509016567/

[14] R. Bedikian, "Understanding latency: Part 1," Leap Motion Blog, Jul 2013. [Online]. Available: http://blog.leapmotion.com/understanding-latency-part-1/

[15] L. Motion, "Leap motion blocks for oculus rift playthrough," YouTube, Feb 2016. [Online]. Available: https://www.youtube.com/watch?v=oZ_53T2jBGg\&feature=emb_title

[16] R. Bedikian, "Understanding latency: Part 2," Leap Motion Blog, Jul 2013. [Online]. Available: http://blog.leapmotion.com/understanding-latency-part-2/

[17] L. R. Brecklin, "Evaluation outcomes of self-defense training for women: A review," Aggression and Violent Behavior, vol. 13, no. 1, p. 60-76, Jan 2008.

[18] "Thumbs up: Hand tracking available on oculus quest this week," Dec 2019. [Online]. Available: https://www.oculus.com/blog/thumbs-up-hand-tracking-now-available-on-oculus-quest/ 